\title{
TREINAMENTO EM SOLUÇÃO DE PROBLEMAS: INTERVENÇÃO EM MULHERES COM DEPRESSÃO*
}

\author{
PROBLEM SOLVING TRAINING: INTERVENTION IN DEPRESSED WOMEN
}

\section{ENTRENAMIENTO EN SOLUCIÓN DE PROBLEMAS: INTERVENCIÓN EN MUJERES CON DEPRESIÓN}

\author{
Nágela Valadão Cade** \\ José Gilberto Prates***
}

Cade NV, Prates JG. Treinamento em solução de problemas: intervenção em mulheres com depressão. Rev Esc Enferm USP 2001; 35(3):223-9.

\section{RESUMO}

Estudo exploratório, descritivo, que objetivou, a partir do Treinamento em Solução de Problemas, realizado em 12 sessões de grupo com mulheres internadas por depressão, avaliar o efeito desta intervenção. Para a avaliação, utilizou-se o Inventário de Depressão de BECK, Inventário de Ansiedade - Estado de SPILBERGER, Lista de Problema de MC KAY, Exercício de simulação por escrito sobre enfrentamento em cinco situações e entrevista sobre a percepção em participarem do grupo. Concluiu-se que o treinamento teve efeitos positivos sobre as pacientes, tanto pela técnica, como pela comunicação estabelecida e pelos fatores terapêuticos grupais que surgiram neste cenário.

PALAVRAS-CHAVE: Depressão.Intervenção de enfermagem.Psicoterapia de grupo.

\section{Saúde da Mulher ABSTRACT}

This is an exploratory and descriptive study which has aimed from the Problem Solving Training accomplished in 12 group sessions with women admitted to hospital suffering from depression, to evaluate the effect of this intervention. BECK Depression Scale, SPILBERGER Anxiety State Scale, MCKAY Problem List, a Written Simulation Exercises about coping in five situations and an interview about the perception of group participation were used for the assessment of the patients. It was concluded that the training had positive effects about the women as for the technique used as for the communication established and for group therapeutic factors which appeared in this scenery.

KEYWORDS: Depression.Nursing intervention.Psychotherapy group.

\section{Women's health. RESUMEN}

Estudio exploratorio descriptivo que objetivô, después del Entrenamiento en solución de problemas realizado en 12 sesiones de grupo con mujeres internadas en hospital por depresión, valuar el efecto de esta intervención. Para la valuación, fueron utilizados el Inventario de depresión de Beck, Inventario de ansiedad de Spilberger, Lista de problema de McKay, Ejercicio por escrito sobre afrentaiento (coping) en cinco situaciones y entrevista sobre compresión de la participación del grupo. La conclusión fue que el entrenamiento tuvo efectos positivos sobre las pacientes, tanto pela tecnica, como pela comunicación establecida y pelos factores terapêuticos del grupo que surgiron en este escenario.

PALABRAS-CLAVE: Depresión. Intervención de enfermeria. Entrenamiento en solución de problemas.

Trabalho apresentado como requisito para conclusão do Curso de Terapia Cognitivo-Comportamental, Faculdade de Medicina da USP, Instituto de Psiquiatria, Ambulatório de Ansiedade (AMBAN).

** Docente do Departamento de Enfermagem da Universidade Federal do Espírito Santo. Mestre em psicologia e doutoranda da Escola de Enfermagem da Universidade de São Paulo - SP.

*** Enfermeiro do Instituto de Psiquiatria da Faculdade de Medicina da USE Especialista em saúde mental. 


\section{INTRODUÇÃO}

Estima-se que 15 a $20 \%$ da população adulta, em algum momento de suas vidas, apresentam sintomatologia depressiva, sendo as mulheres acometidas duas vezes mais em relação aos homens ${ }^{(1)}$.

A depressão é uma doença de causa multifatorial, envolvendo fatores biológicos (neurotransmissores), históricos (depressão e alcoolismo na família), ambientais (situações de conflito, apoio social deficiente, choque emocional) e psicológicos, Caracteriza-se por um quadro de tristeza, choros periódicos, com sentimentos de culpa e decepção com os demais. Pode evoluir com manifestação de irritação, tensão e ansiedade. Em estágio mais elevado, impossibilita o indivíduo de realizar suas atividades diárias, pois há um desinteresse visível e falta de energia ${ }^{(1)}$.

Outros sintomas incluem a baixa concentração, insônia, falta de apetite, apatia sexual, sentimento de desesperança e pensamento em suicídio. Este quadro pode regredir em um espaço de 03 a 06 meses, mesmo sem nenhuma forma de tratamento, porém, com freqüência, há recaídas e 15 a $20 \%$ dos deprimidos cronificam, sendo que o tratamento não farmacológico é importante na diminuição ou eliminação das

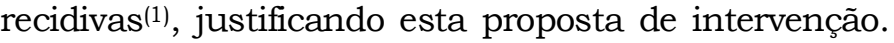
Em casos graves, com ideação suicida, a internação torna-se necessária.

A Terapia Cognitivo-Comportamental utiliza varias técnicas e métodos em pacientes deprimidos. Entre alguns utilizados em grupo, encontrou-se: racionalização de autoconceitos negativos; estimulação de funcionamento como um suporte social (2); aumento de atividades que causam prazer; comunicação assertiva; solução de problemas e planejamento sobre como manipular os sintomas depressivos (03); monitorização das emoções negativas e respectivos pensamentos $\operatorname{associados}(4)$.

Dentro da abordagem citada, escolheu-se aplicar o Treinamento em Solução de Eroblema, segundo o referencial de D'Zurilla, Goldfried(5) adaptado por Nezu, Nezu(6). Para D'Zurilla, Goldfreid(5), este é um processo que faz emergir uma grande quantidade de respostas alternativas para lidar com uma situação problema as quais possibilitam a escolha de respostas mais adequadas e a redução das emoções negativas.

Em pesquisa clínica, esse treinamento tem se mostrado satisfatório na redução do nível de depressão $(7,8)$, sendo a melhora dos sintomas depressivos associada a uma avaliação mais acurada do problema, à maior efetividade na solução do mesmo e à modificação da orientação do locus de controle externo para o interno, permitindo que o indivíduo se coloque mais positivamente frente às suas dificuldades e amplie sua habilidade em resolvê-las.
Este é um estudo exploratório, descritivo, que a partir da aplicação do Treinamento em Solução de Problemas em mulheres deprimidas, objetiva:

a)caracterizar os sujeitos quanto aos problemas vivenciados no dia-a-dia, número e tempo de internação, diagnóstico médico e tratamento;

b)identificar o estado de ansiedade, nível de depressão e estratégias de enfrentamento utilizadas em situações relacionadas com a depressão, antes e depois da intervenção proposta aplicada em grupo;

c)avaliar os efeitos desta intervenção segundo a percepção da clientela.

\section{METODOLOGIA}

\section{Sujeitos}

O grupo foi formado por pacientes do sexo feminino de uma unidade de internação psiquiátrica de um hospital governamental da cidade de São Paulo, tendo a depressão grave como diagnóstico principal ou fase do transtorno afetivo.

\section{Procedimento}

Contactou-se o médico responsável para confirmação diagnóstica e parecer quanto à viabilidade de a paciente participar do Treinamento em grupo. Após, realizava-se entrevista com a paciente, para esclarecer sobre o trabalho a ser realizado e obter o consentimento pós- informado para participarem, tanto do grupo, como da pesquisa.

Optou-se por manter um grupo aberto, homogêneo, com 03 a 06 mulheres em cada sessão. Eram sessões semanais de uma hora, perfazendo um total de 12 sessões, iniciadas em 24 de agosto e terminadas em 01 de dezembro de 1999. Houve a presença de um co-terapeuta do sexo masculino em todas as sessões e de um supervisor para discussão da dinâmica de grupo.

Utilizaram-se as 05 etapas do Treinamento em Solução de Problemas proposto por Nezu, Nezu (6), que foram: a) definição do problema; b) compreensão do problema; c) preparação para a ação, d) tomada de decisão; e) implementação da estratégia escolhida e avaliação.

\section{Instrumentos}

Com o objetivo de identificar o nível de gravidade dos sintomas, utilizou-se o Inventário de Depressão de BECK e o Inventário de Ansiedade Estado de SPILBERGER, após a primeira sessão e, posteriormente, para os sujeitos que continuavam no grupo, após a $3^{\mathrm{a}}$, a $4^{\mathrm{a}}$ ou a $5^{\mathrm{a}}$ sessão. 
A interpretação da pontuação obtida nos instrumentos constituiu-se para o Inventário de Depressão de BECK < 10 pontos: depressão mínima; 10-18 pontos: leve a moderada; $19-29$ pontos: moderada a grave e 30-63 pontos: depressão grave. No Instrumento de Ansiedade-Estado de SPILBERGER, 20-34 pontos: baixa ansiedade; 35-49 pontos: moderada; 50-64 pontos: elevada e 65-80 pontos: ansiedade muito elevada (9).

O levantamento dos problemas de vida percebidos pela clientela foi realizado por meio da Lista de Problemas de Mc Kay, Davis, Fanning(10). Ainda, elabororou-se um Exercício de Simulação por Escrito, de múltipla escolha, com 05 situações de vida relacionadas à depressão, solicitando-se que marcassem uma a três opções de enfrentamento que mais representassem o modo de agirem frente aquelas situações. Esse exercício foi preenchido na fase de prégrupo e após a $11^{\circ}$ sessão, quando toda a técnica de resolução de problemas já havia sido exposta e trabalhada.

Elaborou-se, também, um instrumento semiestruturado com questões fechadas destinadas à caracterização dos sujeitos e com questões abertas, abordando a percepção sobre o Treinamento: 1) 0 que significou participar deste grupo?; 2) 0 grupo trouxevantagens, desvantagens ou nada modificou em sua vida?; Quais foram as vantagens ou desvantagens?; 3) Tem alguma coisa que lhe fará falta no grupo?

\section{APRESENTAÇÃo E DISCUSSÃo DOS RESULTADOS}

Nove pacientes participaram do grupo, porém, nem todas freqüentaram todas as sessões e preencheram os instrumentos para coleta de dados. Portanto, para análise dos dados, consideraramos as pacientes que freqüentaram quatro (04) ou mais sessões, exceto a paciente Judite, que não retornou ao grupo após alta hospitalar, ficando os instrumentos incon-clusivos, e a paciente Jurema, que estava em programa de eletroconvulsoterapia e sem condições de preencher os instrumentos. Os problemas referidos por essas duas pacientes, bem como os comportamentos de enfrentamento relatados na fase pré grupo serão descritos em conjunto com as demais pacientes.

Os dados caracterizando o quadro patológico e o tratamento podem ser vistos na TABELA 1 e os niveis de depressão e ansiedade em diferentes momentos da coleta de dados na TABELA 2.

Tabela 1- Relação das pacientes com dados referentes ao diagnóstico médico, número de internações anteriores, e tratamentos utilizados. São Paulo, Instituto de Psiquiatria - do HC da FMUSP, 1999.

\begin{tabular}{|c|c|c|c|c|}
\hline Paciente & Diagnóstico médico & $\begin{array}{l}\text { número } \\
\text { internação } \\
\text { anterior }\end{array}$ & $\begin{array}{l}\text { eletrocon- } \\
\text { vulso- } \\
\text { terapia }\end{array}$ & Medicamentos usados durante internação \\
\hline Denise & $\begin{array}{l}\text { Depressão } \\
\text { recorrente }\end{array}$ & 13 & não & $\begin{array}{l}\text { Benzodiazepínico - Antidepressivo-IRS } \\
\text { Antidepressivo-IMAO 2- Antipsicótico }\end{array}$ \\
\hline Tereza & depressão & 02 & não & $\begin{array}{l}\text { Benzodiazepínico -Antidepressivo tricíclico } \\
\text { Anti histamínico }\end{array}$ \\
\hline Jurema & Depressão psicótica & 08 & $\operatorname{sim}$ & Benzodiazepínico - Antipsicótico \\
\hline$\overline{\text { Carla }}$ & Depressão & 0 & não & Benzodiazepínico - Antidepressivo tricíclico \\
\hline Noemia & $\begin{array}{l}\text { Transtorno afetivo } \\
\text { bipolar }\end{array}$ & 0 & não & $\begin{array}{l}\text { Benzodiazepínico - Antidepressivo tricíclico } \\
\text { Estabilizador do humor - Antidepressivo- } \\
\text { ISRS } 3\end{array}$ \\
\hline Judite & $\begin{array}{l}\text { Depressão e } \\
\text { transtorno de } \\
\text { personalidade }\end{array}$ & 0 & não & $\begin{array}{l}\text { Benzodiazepínico -Antidepressivo tricíclico } \\
\text { Antidepressivo-ISRS }\end{array}$ \\
\hline
\end{tabular}

Em pacientes com quadro de depressão grave, esperam-se escores elevados nos instrumentos de medida de depressão e de ansiedade, o que é compativel com os dados da TABELA 2. Observa-se em Denise, Noemia e Judite melhora destes sintomas depressivos.

\footnotetext{
IRS: inibidores da recaptação da serotonina.

IMAO: inibidores da monoaminoxidase.

3 ISRS: inibidores seletivos da recaptação da serotonina.
} 
Tabela 2 - Relação das pacientes com a data de internação e com os escores obtidos nos Instrumentos de Depressão de BECK e Ansiedade-estado de SPILBERGER, com respectivas datas e significado. São Paulo, Instituto de Psiquiatria do HC da FMUSP, 1999.

\begin{tabular}{|c|c|c|c|c|c|c|}
\hline \multirow{2}{*}{$\begin{array}{l}\text { Paciente e } \\
\text { internacão }\end{array}$} & \multicolumn{3}{|c|}{ Inventário Depressão de BECK } & \multicolumn{2}{|c|}{ Inventário } & Ansiedade-estado \\
\hline & Data & Pontuação & Significado & Data & Pontuação & Significado \\
\hline Denise & $24 / 08$ & 41 & grave & $24 / 08$ & 73 & muito elevada \\
\hline \multirow[t]{4}{*}{ 06/08/99 } & $14 / 09$ & 49 & grave & $14 / 09$ & 65 & elevada \\
\hline & $05 / 10$ & 39 & grave & $05 / 10$ & 51 & elevada \\
\hline & $09 / 11$ & 35 & grave & $09 / 11$ & 54 & elevada \\
\hline & $25 / 11$ & 32 & grave & $25 / 11$ & 50 & elevada \\
\hline Tereza & $03 / 11$ & 44 & grave & $03 / 11$ & 64 & elevada \\
\hline $03 / 10 / 99$ & $25 / 11$ & 39 & grave & $25 / 11$ & 58 & elevada \\
\hline $\begin{array}{l}\text { Jurema } \\
20 / 10 / 99\end{array}$ & $04 / 11$ & 34 & grave & $04 / 11$ & 72 & muito elevada \\
\hline \multirow{2}{*}{$\begin{array}{l}\text { Carla } \\
05 / 10 / 99\end{array}$} & $08 / 10$ & 25 & moderada-grave & $08 / 10$ & 55 & elevada \\
\hline & $01 / 12$ & 22 & moderada-grave & $01 / 12$ & 53 & elevada \\
\hline Noemia & $24 / 08$ & 46 & grave & $24 / 08$ & 68 & muito elevada \\
\hline \multirow[t]{2}{*}{$20 / 08 / 99$} & $\begin{array}{l}15 / 09 \\
04 / 10\end{array}$ & $\begin{array}{l}46 \\
32\end{array}$ & $\begin{array}{l}\text { grave } \\
\text { grave }\end{array}$ & $\begin{array}{l}15 / 09 \\
04 / 10\end{array}$ & $\begin{array}{l}64 \\
52\end{array}$ & $\begin{array}{l}\text { elevada } \\
\text { elevada }\end{array}$ \\
\hline & $26 / 11$ & 24 & moderada-grave & $26 / 11$ & 61 & elevada \\
\hline Judite & $24 / 08$ & 20 & moderada-grave & $24 / 08$ & 59 & elevada \\
\hline $16 / 08 / 99$ & $15 / 09$ & 16 & leve-moderada & $15 / 09$ & 53 & elevada \\
\hline
\end{tabular}

Das 21 áreas de comprometimento pela depressão contidas no Inventário de Beck, as mais citadas nos diferentes momentos da coleta de dados foram: estar sendo punida (08 respostas); dificuldade em tomar decisão (06); desânimo (06); tristeza (05) e sentir-se culpada (05).

No que diz respeito aos problemas do cotidiano, citaram em maior freqüência os problemas de ordem fisica e psicológica, que foram: transtornos de sono (05 respostas), nervoso (05), preocupar-se com facilidade (04), ausência de motivação (04), cansaço freqüente (03), transtorno de peso (03) e efeitos colaterais da medicação (03). Ainda citaram os problemas relacionados aos gastos financeiros inesperados (04), medo de perder o trabalho (03), dificuldade em iniciar e manter uma conversa na esfera de relacionamento social (03) e os problemas com familiares do tipo desentendimento com marido (03), pais, irmãos e outros (03).

Os problemas listados fazem supor que a depressão tem um impacto importante na vida dessas pacientes, tanto pela sintomatologia como pelas conseqüências que a doença pode provocar em suas vidas.

Quanto ao exercício de múltipla escolha, compreendeu 05 questões. A primeira dizia respeito ao sentir-se cansada para realizar as tarefas diárias e as respostas mais citadas, na fase de pré treinamento por 06 pacientes, foram: ficam preocupadas com a situação (04 respostas); realizam as atividades totalmente (03) ou pela metade (02). Quatro pacientes intercalaram essas atitudes mais passivas frente à situação com algum comportamento mais atuante, como fazer lista de prioridades de tarefas diárias (01), procurar ajuda profissional (01), intercalar tarefa com períodos de repouso para melhorar o cansaço $(01)$ e conversar com familiares e colegas para desabafar ou obter idéias (01).

Nessa questão, prevaleceram respostas evidenciando que o cansaço é um sintoma que muito as incomoda e preocupa, mas que pouco fazem para modificar a situação, e segundo Beck AT, Rush AJ, Shaw $\mathrm{BF}$, Emery $\mathrm{G}^{(11)}$, um dos sintomas-alvo comportamentais da depressão é a passividade frente aos problemas.

A segunda questão dizia respeito a não estar conseguindo executar todas as tarefas delegadas pela chefia. Como somente três delas trabalhavam, foi solicitado que imaginassem a situação e, assim, surgiram as seguintes respostas: programam as atividades a serem executadas (04 respostas), procuram familiares ou colegas para conversarem sobre o assunto (04) e ficam preocupadas com a situação (03). Uma paciente adia, ao máximo, pensar sobre a situação e outra procura conversar com a chefia sobre as dificuldades que vem apresentando.

Entende-se que as questões de trabalho as preocupam, mas contrariamente à situação anterior, apresentam comportamentos mais ativos e busca de apoio. Dessas pacientes, 03 estavam sem trabalho devido à doença, e somente 01 tinha vínculo empregatício.

A terceira pergunta dizia respeito ao que faziam frente ao sentimento de raiva e ressentimento com o companheiro e, neste caso, responderam: procuram conversar com o companheiro (03 respostas) ou pensam nessa possibilidade (02); rezam (03); conversam com familiares (02); e pensam em separar-se (02). 
A quarta questão dizia respeito ao sentir-se sozinha, sem amigos, e as respostas foram: tentam ajudar as pessoas para formar vinculo (03); procuram alguém que já conhecem (02) ou pensam na possibilidade de fazê-lo (02) e rezam (02).

A última pergunta referia-se à situação de vida que a paciente deveria negar o empréstimo de um objeto a uma vizinha, e as respostas foram muito variadas e, por vezes, contraditórias, mas uma adia o empréstimo, outra pede opinião de outros antes de emprestar, uma nega o pedido, outra empresta o objeto, não se importando, e outra nega o pedido e volta atrás emprestando o objeto.

Esse exercício mostrou que as pacientes ficam impotentes frente aos sintomas da depressão, mas agem de forma mais confrontiva em relação aos problemas de trabalho, conflito com o cônjuge, ficou ainda, entendido que o suporte social é uma coisa importante que tentam preservar.

Como somente quatro pacientes preencheram todos os critérios para coleta de dados, achamos pertinente apresentar seus dados individualmente.

DENISE: 43 anos, $2^{\circ}$ grau incompleto, casada, com uma filha de 18 anos. Atualmente, desempregada. Desconhece a renda familiar.

Freqüentou todas as sessões e seu problema foi o escolhido para iniciar as etapas do Treinamento. Das quatro estratégias traçadas pelo grupo para minimizar o problema, duas foram colocadas em ação, tendo uma avaliação satisfatória quanto à tomada de iniciativa e melhora do problema que a afligia, mostrando, assim, que o objetivo proposto foi atingido.

Como problemas, citou a dificuldade em expressar sentimentos, os sintomas da depressão, o efeito colateral da medicação, o preço dos remédios, o medo de perder o emprego, e os aspectos ligados à baixa habilidade social, como não conseguir dizer não ou defender o que deseja.

Para Beck AT, Rush AJ, Shaw BF, Emery G(11), o deprimido, em todos os segmentos da vida, apresenta deficiência na esfera social, estando prejudicado o uso da habilidade em relacionar-se no interpessoal.

Quanto as cinco questões do Exercício de simulação analisadas em dois diferentes momentos (pré e pós-grupo), evidenciou-se que apresenta uma postura passiva frente ao sintoma de cansaço e indecisão ao defender o que deseja. Quanto ao marido, referiu que tenta conversar com ele, pois, atualmente, este tem sido a pessoa mais significativa em termos de apoio e ajuda nos aspectos ligados à doença, já que não tem amigos, e os familiares encontram-se afastados.

Constatou-se mudança significativa na esfera do trabalho após o Treinamento, pois ao responder o instrumento no segundo momento, decidiu expor as dificuldades para a chefia e colegas e solicitar-lhes ajuda, ao invés de somente ficar preocupada.

Sua maneira de enfrentamento direciona-se para a negação de envolver-se com seus problemas ou para o adiamento em resolvê-los, preocupando-se de forma excessiva, o que pode ser entendido como um dos sintomas-alvo de ordem emocional da depressão, pois o deprimido tem falta de motivação geral e não acredita que se beneficiará com as atividades(11).

Quanto aos instrumentos de medida, evidenciouse uma redução na pontuação (menos 23 pontos na ansiedade e 09 pontos na depressão), evoluindo de ansiedade "muito elevada" para "elevada", mas a depressão manteve-se "grave". Evidenciou-se que as nos primeiros dois instrumentos respondidos, 12 áreas das 21 do instru-mento estavam comprometidas em grau máximo, enquanto nos dois últimos instrumentos, somente duas áreas foram observadas (tristeza e insatisfação).

Quanto à percepção da estratégia adotada, avaliou o grupo como "proveitoso (...) pois tentam mostrar como lidar com os problemas no sentido de amenizá-los (...) já tem a doença e ainda mais os outros problemas". Relata que sentirá falta dos componentes do grupo como um todo e das sugestões dadas.

TEREZA: 56 anos, solteira, sem filhos, vive com uma irmã. Costureira autônoma, porém atualmente, encontra-se incapacitada para o trabalho. Concluiu o primeiro grau. Sem renda própria.

Entre os problemas citados, estão os sintomas físicos e psicológicos da depressão, como alteração do sono, peso, cansaço, nervoso, preocupação e dificuldade em relacionar-se socialmente, como timidez, iniciar e manter conversa e recusar pedidos.

No Exercício de Simulação, procurou intercalar repouso com as atividades no sentido de melhorar o cansaço. Em relação trabalho, não toma nenhuma iniciativa frente às dificuldades e quanto às demais três questões, foi evidenciado, melhora no enfrentamento, considerando o momento pré e pós grupo. Quanto ao companheiro, procura conversar em vez de rezar; tenta conversar com familiares e ser prestativa como forma de estabelecer vínculo e toma decisão em relação a emprestar algo de sua casa. As respostas do primeiro instrumento mostraram mais atitudes para aliviar a angústia, como rezar e menos voltadas para a solução dos problemas.

Posicionou-se sobre o grupo como um lugar para identificar seu problema de depressão com outras pessoas e pensar sobre as colocações verbais dos elementos do grupo.

Como vantagens, refere que aprendeu sobre a depressão porque não sabia o que vinha a ser a doença e pôde se comunicar mais, já que é muito tímida, "talvez me soltando possa encontrar o que está dentro de mim e ajudar o problema da depressão": Disse, ainda, que gosta de estar no grupo porque "passam uma coisa boa para a gente e me alivia. Só em ouvir as pessoas se expressarem fico aliviada".

Quanto aos instrumentos de medida de ansiedade e depressão, houve redução pequena (06 e 05 pontos respectivamente) na pontuação obtida, 
apesar de a paciente mostrar-se com facies alegre e estar mais cuidadosa com a aparência física.

NOEMIA: 48 anos, viúva, sem filhos, cozinheira de festas ou restaurante, desempregada. Morava sozinha, mas pelas idéias suicidas e dificuldade financeira, foi morar com a irmã e três sobrinhos adultos após a última internação. Concluiu o primeiro grau.

Os comportamentos de enfrentamento frente às situações do Exercício de Simulação foram os mesmos antes e depois do treinamento em três questões (sobre o cansaço, dificuldades no trabalho e sentimento de estar só), com atitudes passivas e ativas, concomitantemente, para um mesmo problema. Apresentou resposta contraditória na questão sobre o companheiro e quanto a emprestar algum material.

Os problemas citados abrangeram mais esferas além da doença depressiva, pois além das queixas relacionadas com a depressão, pontuou dificuldades financeiras e problemas familiares (conflito, desentendimento e mágoa).

O nivel de ansiedade e depressão modificou significativamente de ansiedade "muito elevada" para "elevada" e depressão "grave" para "moderada a grave". O último instrumento foi preenchido após 50 dias de alta hospitalar.

Posicionou-se sobre o grupo como um local que possibilitou ver "que não é só a gente que tem problemas. Todos ali têm um problema sério". Como vantagens, citou que o grupo a ajudou na auto-estima por não sentir-se única com suas dificuldades. Sendo muito fechada e reservada, teve a oportunidade de "poder se abrir um pouco, dar opiniões, falar o que a gente sente". Sentirá falta da amizade formada e do conhecimento que os elementos do grupo lhe proporcionaram.

CARLA: 27 anos, recém-separada, $6^{\circ}$ mês de gestação, com dois filhos de 04 e 07 anos, prendas do lar. Possui o $2^{2}$ grau incompleto e recebe pensão do ex- marido.

Como problemas, citou em maior freqüência os desentendimentos e discussões com familiares e companheiro. Estava em uma fase de crise, pois todo o processo de separação era muito recente, o que ela atribuía como causa da depressão e motivo da internação. Chorava sempre ao falar sobre o assunto, mas estava disposta a fazê-lo. Referiu, ainda, problemas psicológicos, que podem estar associados à depressão, como nervoso, preocupação e dificuldade em alcançar os objetivos e insuficiência de dinheiro.

Apesar de aparente melhora no humor, na aparência física e sem choro constante, a pontuação do instrumento de medida de ansiedade e depressão só reduziu 02 a 03 pontos, mantendo-se "elevada".

Quanto ao Exercício de Simulação, citou realizar comportamentos ativos na primeira e na segunda questão, mobilizando seus próprios recursos de enfrentamento. Praticamente, não houve mudança no enfrentamento, considerando-se pré e pós-grupo. Mesmo após alta hospitalar, continuou vindo regularmente às sessões e relatou que participar do grupo "está sendo uma escola, pois está aprendendo as coisas do coração. A gente tem que aprender a lidar com as coisas do coração também, igual aprendemos matemática, português. A vida não ensina tudo".

Como vantagens, referiu que pôde ver que outras pessoas tinham problemas semelhantes e que da mesma forma que somos ajudado por outros, também ajudamos, ficando explicitada a função de suporte social desempenhada pelo grupo. Outra vantagem apontada foi a orientação de profissionais que tinham competência para cuidar delas, o que era diferente de conversar com amigos. Também, que sentirá falta dos componentes do grupo, das conversas e do aconchego.

Todas referiram que irão sentir falta das companheiras do grupo e das conversas.

\section{CONSIDERAÇÕES FINAIS}

Este trabalho de pesquisa apresentou algumas limitações, sendo uma delas, o fato da depressão e dos efeitos de algumas medicações interferirem no raciocínio e, assim, influenciarem nas respostas das pacientes, visto que o Treinamento propunha ensinar uma técnica que ajudasse a pessoa a reconhecer um problema, traçar e avaliar estratégias de ação e realizá-las.

A depressão foi apontada como o principal problema na vida dessas mulheres, exceto por Carla, que teve, nessa internação, o primeiro contato com a doença, atribuindo-a à crise conjugal.

Em alguns momentos, no grupo, relataram sentirem-se discriminadas por terem uma doença psiquiátrica, ou por vezes, os familiares não davam importância as suas queixas "(...) isto é frescura e coisa de rico". Manifestaram também o intenso desconforto dos sintomas depressivos, como tristeza, falta de motivação, ausência total de prazer, não conseguir trabalhar e vontade de morrer.

Outro aspecto observado foi que as pacientes apresentavam baixa habilidade social. As pacientes Noemia, Tereza, e Denise no encerramento do Treinamento, fizeram referência a gostarem do grupo porque houve oportunidade de expor os sentimentos, dar opiniões, posicionar-se sobre algo, defender o que pensam e "comunicar-se", possibilitando, assim, melhorar suas dificuldades..

Quanto às respostas aos instrumentos de medida de ansiedade e depressão, pode ter sido influenciada pela da medicação anti-depressiva, pois todas as pacientes tiveram modificação na dose ou tipo de medicação ingeridas enquanto participavam do grupo, e espera-se que à medida que o tempo de tratamento avança, ocorra melhora dos sintomas depressivos. Os resultados mostraram que houve redução da pontuação nos instrumentos de ansiedade e depressão em todas as pacientes nos dois instrumentos, e quanto à depressão, houve redução das áreas sintomáticas 
comprometidas em grau máximo, mas, em poucos momentos, houve diminuição no nivel de depressão e ansiedade, segundo a padronização dos escores e respectivo significado clínico.

No Exercício de Simulação por escrito, observou-se alguma modificação nas atitudes de Denise e significativas em Tereza, considerando-se o momento pré e pós grupo.

Pode-se, também, pensar que os comportamentos de enfrentamento sofrem influência de outros fatores, que não são só a aprendizagem de uma técnica, como, por exemplo, o traço de personalidade, locus de controle e outras caracteristicas do individuo ${ }^{(12)}$

Outros questionamentos que levantamos constituiu-se no comprometimento cognitivo causado pela depressão ou pela medicação que poderia estar prejudicando a assimilação da técnica. Isto pôde ser visto quando era iniciada uma sessão, e as pacientes apresentavam dificuldades em lembrar-se da sessão anterior. Beck AT, Rush AJ, Shaw BF, Emery G(11) comenta sobre a dificuldade de concentração e memória como sintomas da depressão.

No entanto, avaliou-se que este tipo de Treinamento trouxe alguma mudança nessa clientela no que diz respeito à percepção mais detalhada do que vem a ser um problema e nas possibilidades e capacidades de enfrentamento, e os questionamentos cita-dos podem ser um ponto de partida para estudos posteriores sobre a efetividade do Treinamento em Solução de Problemas com pacientes depressivos.

Por outro lado, as pacientes explicitaram alguns beneficios com o grupo que entendemos serem devido aos fatores terapêuticos que permeiam a dinâmica grupal.

Ocorreu com o grupo do estudo fatores citados por Vinogradow, Yalom (13) como universalidade quando os elementos do grupo identificaram aspectos comuns entre si, sendo, neste caso, o elo de ligação a doença depressiva e toda sua sintoma-tologia. Percebia-se que as pacientes ficavam surpresas ao defrontarem-se com os mesmos sintomas e dificuldades.

Ainda fatores observados ou reconhecidos pelos componentes foram o altruísmo quando os componentes do grupo ofereceram ajuda aos demais contribuindo com idéias; catarse (expressão de emoções enrustidas) visualizada em Denise ao falar do marido e da filha e em Carla sobre o marido; estimulação da esperança quanto aos problemas terem solução; repasse de informação pelo terapeuta, de acordo com as situações que surgiram no grupo. Dentre algumas oportunidades terapêuticas, foram abordados sobre a possibilidade da cognição e dos pensamentos negativos interferirem em nossas ações, inibindo-as; as situações, que aparentemente apresentam pouca ou nenhuma solução, quando têm oportunidade de reflexão, estimulam as diversidades de opções; as ações, aparentemente, fáceis, devem ser avaliadas pensando nas possiveis conseqüências desta sobre nossa vida e nas de pessoas significativas; e informações sobre depressão que possibilitaram Tereza compreender seu quadro clínico.

Conclui-se que o Treinamento em Solução de Problemas apresentou efeitos satisfatórios, pela técnica em si, pelo apoio, pelo processo comunicativo que se estabeleceu entre os membros, favorecendo as pacientes com menos habilidade social e pelos fatores terapêuticos pertinentes à dinâmica grupal.

\section{REFERÊNCIAS BIBLIOGRÁFICAS}

(1) Hawton K, Salkovskis PM, Kir J, Clark DM. Terapia cognitivo-comportamental para problemas psiquiátricos. um guia prático. São Paulo:Martins Fontes, 1997.

(2) Koder DA,Brodaty H, Anstey K. Cognitive therapy for depression in the elderly. Int $\mathrm{J}$ Geriatr Psychiatry 1996; 11:97-107.

(3) Peterson AL,Halstead TS. Group cognitive behavior therapy for depression in a community setting: a clinicar replication series. Behav Ther 1998;29:3-18

(4) Thompson LW. Cognitive-behavioral therapy and treatment for late-life depression. J Clin Psychiatry. 1996;57: 29-37)D'Zurilla TJ, Goldfried MR. Problem solving and behavior modification. J Abnorm Psycho! 1971;78(1): 107-26.

(5) Nezu AM, Nezu CM. Treinamento em solução de problemas. In Caballo VE. Manual de técnicas de terapia e modificação do comportamento. São Paulo:Santos, 1999. cap. 22, p. 47193.

(6) Nezu AM. Efficacy of a problem-solving therapy approach for unipolar depression. J Cons Clin Psycho! 1986;54(2): 196-202.

(7) Nezu AM, Perri MG. Social problem-solving therapy for unipolar depression: na initial dismantling investigation. J Cons Clin Psychol 1989;57(3):408-13.

(8) Gorenstein C, Andrade L. Inventário de depressão de Beck: propriedades psicométricas da versão em protuguês. Rev Psiq Clin 1998;25(5 ed esp):245-50

(9) Mc Kay M, Davis M, Fanning P Técnicas cognitivas para el tratamiento del strés. Barcelona: Martinez Loca, 1985. La solución de problemas. cap 5, p. 71-87.eck AT, Rush AJ, Shaw BF, Emery G. Terapia cognitiva da depressão. Porto Alegre:Artes Médicas, 1997.

(10) Lazarus RS, Folkman S. Estresse, appraisal and coping. New York: Springer, 1984.

(11) Vinogradow S, Yalom ID. Gula breve de psicoterapia de grupo. Barcelona: Paidós, 1996.

Artigo recebido em 22/02/00
Artigo aprovado em 01111101

